\title{
Dissociation of perceptual and motor inhibitory processes in young and elderly participants using the Simon task
}

\author{
SOPHIE GERMAIN ${ }^{1}$ AND FABIENNE COLLETTE ${ }^{1,2}$ \\ ${ }^{1}$ Cognitive and Behavioral Neuroscience Center, University of Liège, Liège, Belgium \\ ${ }^{2}$ Cyclotron Research Center, University of Liège, Liège, Belgium
}

(Received February 14, 2008; Final Revision June 20, 2008; Accepted July 1, 2008)

\begin{abstract}
Deficits in inhibitory abilities are frequently observed in normal aging. However, few studies have explored the generality of these deficits in a single group of participants. Here, we used an adaptation of the Simon task to differentially assess perceptual and motor inhibition using the same stimuli and task design and to determine whether these processes use separate or shared cognitive resources. We were interested in determining whether (1) normal aging is associated with the use of separate (as previously evidenced in young participants) or similar cognitive resources to perform perceptual and motor inhibition tasks; (2) older participants present a specific impairment in one of these two processes. Analyses of reaction times indicated that motor and perceptual inhibitory processes share some cognitive resources and both are impaired in normal aging. These results can be interpreted by considering that a dedifferentiation process is responsible for the inhibitory deficits presented by older participants. (JINS, 2008, 14, 1014-1021.)
\end{abstract}

Keywords: Aging, Conflict, Inhibitory control, Motor inhibition, Perceptual inhibition, Dedifferentiation process

\section{INTRODUCTION}

Inhibition is a basic aspect of cognitive and emotional functioning, which is involved in the performance of numerous tasks and processes. Changes in inhibitory functioning have been reported across the entire life span (Harnishfeger, 1995; Harnishfeger \& Bjorklund, 1993); and normal aging has frequently been found to be associated with a decrease in inhibitory abilities. Indeed, difficulties suppressing an overlearned response or an irrelevant dimension of the stimulus, respectively, have been observed with the Stroop task (Dulaney \& Rogers, 1994; Houx et al., 1993; Spieler et al., 1996) and the negative priming paradigm (Hasher et al., 1991; Kane et al., 1994; Stoltzfus et al., 1993). Additionally, age-related differences in inhibition of irrelevant semantic information (Andrés \& Van der Linden, 2000; Connelly et al., 1991; Hartman \& Hasher, 1991), and difficulties suppressing inappropriate, but prepotent, motor responses have

Correspondence and reprint requests to: Fabienne Collette, Neuropsychology Unit, University of Liège, Boulevard du Rectorat 3 (B33), 4000 Liège, Belgium. E-mail: f.collette@ulg.ac.be also been reported (Butler et al., 1999; Nielson et al., 2002). Finally, a decreased ability to suppress irrelevant information in working and episodic memory tasks has also been observed (Andrés et al., 2004; Zacks et al., 1996).

However, aging has not been systematically observed to have a negative effect on inhibition abilities. This absence of deficits was sometimes observed on very similar tasks by those who demonstrated impairment (e.g., the Stroop or negative priming tasks). So, a performance similar to that of young participants has sometimes been reported for the Stroop (Kieley \& Hartley, 1997) and negative priming (Connelly \& Hasher, 1993; Langley et al., 1998; Sullivan et al., 1995) tasks. A normal performance was also observed for inhibition of return (Hartley \& Kieley, 1995), antisaccade (Eenshuistra et al., 2004,), go/no-go (Rush et al., 2006), and dichotic listening tasks (Murphy et al., 1999). Moreover, the few studies that administered several inhibitory tasks to the same groups of young and older participants also demonstrated a specific impairment of some processes associated with the preservation of the others (Andrés et al., 2008; Charlot \& Feyereisen, 2005; Kramer et al., 1994; see, however, Belleville et al., 2006). For example, in the Charlot and Feyereisen's study, the effects of aging on working 
memory are weaker on the access than on the suppression functions.

Taken as a whole, these results indicate that not all inhibitory processes are affected by normal aging. One criticism that can, nevertheless, be made of these studies is that they explored inhibition without any reference to the theoretical frameworks proposed in the literature (e.g., Dempster \& Corkill, 1999a,b; Harnishfeger, 1995; Hasher et al., 1999; Kipp, 2005; Nigg, 2000). Among these frameworks, the proposal by Dempster and Corkill (1999a,b) that there is a distinction between perceptual, motor, and verbal inhibition appears particularly interesting. Indeed, this proposal is theoretically supported by data obtained in young adults with conflict resolution tasks (Nassauer \& Halperin, 2003). Nassauer and Halperin gave young participants several tasks requiring (or not requiring) perceptual and/or motor conflict resolution. Their results showed that the necessity to simultaneously resolve perceptual and motor conflicts leads to an additive (not interactive) effect on reaction times in comparison to the separate resolution of each kind of conflict. According to the limited capacity resources and additive factor models (Sergeant, 1996), this pattern of results suggests that these two processes use distinct cognitive resources such that motor and perceptual inhibition should be considered as separate cognitive processes. As indicated previously, studies that explored perceptual and motor inhibitory processes in older participants lead to contradictory results, with some studies showing impairments but not others. The exploration of these processes with the procedure of Nassauer and Halperin should allow determining whether there exists a specific preservation of one of these processes in normal aging, that will be a supplementary argument in favor of the proposal of Dempster and Corkill.

Moreover, at this time, no study really tackled the question if the lower performance of older participants on a large range of tasks assessing inhibitory functioning may be due to a series of specific deficits or to a less efficient functioning of a general factor. An argument in favour of the intervention of a general factor to explain cognitive changes associated to normal aging is that correlations among different cognitive measures tend to increase with age and that the effect of age on a series of complex cognitive tasks is largely explained by the efficiency of sensorimotor processing (Baltes \& Lindenberger, 1997; Lindenberger \& Baltes, 1994). Similarly, Li et al. (2004) showed that processing robustness in normal aging is largely correlated to performance on cognitive tasks assessing fluid intelligence abilities. Baltes and Lindenberger invoked age-related changes in neurophysiological brain functioning as a common explanation to these data and considered that they reflect the presence of a dedifferentiation process in normal aging.

The aim of the present study is to determine whether the independence of perceptive and motor inhibitory processes found by Nassauer and Halperin (2003) in young participants is preserved during normal aging. Indeed, the presence of independence between these two processes, associated with a lower performance in one (or both) con- flict resolution tasks, will be in agreement with a specificity of inhibitory dysfunction in normal aging. On the contrary, the presence of intercorrelations between performance levels on the two tasks will be indicative of the contribution of a more general factor (e.g., a dedifferentiation process). Therefore, we administered the set of computerized tasks developed by these authors to assess the separability of the two inhibitory processes needed to resolve perceptual or motor conflicts. The two tasks were (1) inhibition of irrelevant stimulus characteristics and (2) inhibition of inappropriate motor responses. The tasks were designed to be independent of verbal ability and to minimize interference due to extraneous stimulus-response modality conflicts. We hypothesised that if older participants use some similar cognitive resources to perform the two inhibitory tasks (due to a dedifferentiation process), we should observe a significant interaction effect when the tasks are presented together in comparison to separate presentations. Furthermore, we were also interested in determining whether perceptual and motor inhibition processes are selectively preserved in normal aging. Indeed, previous studies have explored these two aspects of inhibition in different groups of participants, sometimes obtaining contradictory results (e.g., Butler et al., 1999; Eenshuistra et al., 2004; Kieley \& Hartley, 1997; Spieler et al., 1996).

\section{METHOD}

\section{Participants}

Forty young and thirty-five older adults participated in the study. The young participants ranged in age from 21 to 28 years $(M$ age $=24.15 ; S D=1.69)$ and older adults from 60 to 75 years $(M$ age $=66.69 ; S D=5.22)$. Although young participants had a higher educational level (years of education: younger participants $M=15.97$; $S D=1.31$; older participants $M=14.22 ; S D=2.25 ; t(73)=4.17 ; p<$ $.0001)$, the vocabulary level of older participants was superior [Mill Hill Vocabulary Test (part A and B): young participants $M=36.57 ; S D=3.64$; older participants $M=$ 38.86; $S D=3.61 ; t(73)=-2.72, p=<.01]$. All the older participants scored above 130 on the Mattis Dementia Rating Scale (Mattis, 1976; $M=142.48 ; S D=1.72$ ), which constitutes a cut-off score to discriminate normal aging from dementia (Monsch et al., 1995). All participants were native French speakers with normal or corrected-to-normal vision. They all reported themselves to be in good health. None had a history of significant neurological or psychiatric illness or were currently taking psychoactive medication. The local research ethics committee had approved the study and consent was obtained from each participant.

\section{Material and Procedure}

The tasks described in this study are part of a larger battery that was designed to explore inhibitory functioning in elderly 
persons. All the participants were individually tested in a quiet room in two 1.5-hr sessions that took place over a few days. All the tasks were presented on a laptop with a 15-inch color monitor using E-Prime software version 1.0 (Schneider et al., 2002). Participants were seated in front of the computer at approximately $50 \mathrm{~cm}$ from the screen.

\section{Tasks}

The experimental design was adapted from Nassauer and Halperin (2003) and is composed of six subtests, designed to evaluate the ability to inhibit inappropriate motor responses and/or ignore irrelevant perceptual stimulus characteristics (subtests 3,5,6), or to control for perceptual and motor requirements of the inhibitory tasks (subtests 1, 2, 4; see Figure 1). All responses were made using either a left ("q") or right ("l") response key on a standard AZERTY keyboard. At the beginning of each subtest, participants were instructed to respond as quickly as possible while avoiding errors. The six subtests were always administered in the same order described below.

Subtest 1 consisted of 40 randomized trials in which a left (20 trials) or right (20 trials) pointing arrow appeared in the middle of the screen. Participants are instructed to press either the left or right key depending upon where the arrow is pointing. Subtest 2 involved 40 randomized trials in which a rectangular box appeared randomly either on the left (20 trials) or right (20 trials) side of the computer screen. Participants were instructed to press the key located on the same side as the box. These two subtests did not require inhibition but were used to elicit any tendency to respond following either the direction of the arrows or the location of the stimuli, because both of these characteristics must be inhibited in the next subtests.

In subtest 3, which assesses perceptual inhibition abilities, the 80 trials involved a left (40 trials) or right (40 trials) pointing arrow appearing randomly on either the left (40 trials) or right (40 trials) side of the screen. Participants were asked to ignore the location of the arrow and to press the key on the side indicated by the arrow's direction. Only the 40 items requiring perceptual inhibition (a left pointing arrow located on the right and a right pointing arrow located on the left) were taken into account in our analyses.

In subtest 4 , the material was similar to that used in subtest 1 and consisted of 40 randomized trials in which left (20 trials) or right (20 trials) pointing arrows appeared in the middle of the screen. Participants were, again, instructed to press the key that was on the side to which the arrow was pointing. In subtest 5 , assessing motor aspects of response inhibition, participants were asked to press the key that is opposite to where the centrally located arrow was pointing. This task required motor inhibition because the direction of the arrow did not correspond to the laterality of the response to be produced.

Finally, subtest 6 involved 80 randomized trials in which 20 left-pointing arrows appeared on the left side, 20 leftpointing arrows appeared on the right side, 20 right-pointing arrows appeared on the left side and 20 right-pointing arrows appeared on the right side. The relevant dimension was the arrow's direction; participants were instructed to ignore its location (as in subtest 3 assessing perceptual inhibition). Furthermore, participants were asked to press the key that is opposite to where the arrow was pointing, which required motor inhibition (as in subtest 5). Therefore, some items of subtest 6 simultaneously involved perceptual (ignore location) and motor (press opposite side) conflict. Only these 40 items were taken into account in the analyses.

For all subtests, the stimuli (both arrows and boxes) were displayed for a maximum of 3,000 milliseconds or until the participant responded. Specific instructions and practice trials were administered before the beginning of each subtest.

\section{RESULTS}

We first performed factorial ANOVAs to replicate the dissociation between perceptual and motor conflicts observed

\begin{tabular}{|c|c|c|c|}
\hline & & \multicolumn{2}{|c|}{ Perceptual conflict } \\
\hline & & No & Yes \\
\hline \multirow{2}{*}{ 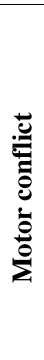 } & No & Compatible response to direction & Compatible response to direction \\
\hline & Yes & Incompatible response to direction & Incompatible response to direction \& location \\
\hline
\end{tabular}

Fig. 1. Specific subtest trials used in calculating mean reaction times for data analyses. Numbers in brackets indicates the number of inhibitory or control trials really used in statistical analyses. Data of subtest 2 are not used in the analyses. 
Table 1. Mean reaction times (milliseconds) and percentage of correct responses in the young and elderly participants

\begin{tabular}{|c|c|c|c|c|}
\hline \multirow[b]{2}{*}{ Subtest } & \multicolumn{2}{|c|}{ Reaction times } & \multicolumn{2}{|c|}{ Response accuracy } \\
\hline & Young & Elderly & Young & Elderly \\
\hline No conflict (subtests $1 \& 4,80$ trials) & $376(5)$ & $521(7)$ & $98.5(0.27)$ & $98.46(0.31)$ \\
\hline Neutral condition (subtest 2, 40 trials) & $312(5)$ & $468(18)$ & $99.18(0.26)$ & $98.35(0.42)$ \\
\hline Perceptual conflict (subtest 3, 40 trials) & $505(9)$ & $705(19)$ & $96.50(0.66)$ & $94.92(1.5)$ \\
\hline Motor conflict (subtest 5, 40 trials) & $422(10)$ & $724(31)$ & $97.25(0.48)$ & $94.85(1.89)$ \\
\hline Perceptual/motor conflict (subtest 6, 40 trials) & $538(12)$ & $834(45)$ & $96.75(0.79)$ & $85.86(3.87)$ \\
\hline Perceptual conflict size* & $129(8)$ & $184(11)$ & & \\
\hline Motor conflict size ${ }^{* *}$ & $45(8)$ & $203(21)$ & & \\
\hline
\end{tabular}

Note. Numbers in parentheses are standard errors. Trials number refers to the number of items used for statistical analyses.

*The perceptual conflict size is calculated by subtracting RTs in the no conflict condition from RTs in the perceptual conflict condition.

**The motor conflict size is calculated by subtracting RTs in the no conflict condition from RTs in the motor conflict condition.

by Nassauer and Halperin (2003) in young participants and to determine whether such a dissociation also exists in older adults. Next, we directly contrasted perceptual and motor inhibitory abilities in young and older participants. In subtests $1,2,4$, and 5 , all presented items were used for statistical analyses. In subtests 3 and 6 , only items necessitating conflict resolution were included in the analyses. All effects were assessed for significance at the $p=.05$ level. The effect size of each analysis was reported as partial eta square for the main effects and the interactions. Partial eta square is generally interpreted as the proportion of variance of the dependent variable that is related to the factor. Traditionally, eta square values of $.01, .06$, and .14 represent small, medium and large effect sizes, respectively (Cohen, 1988).

Reaction time (RT) analyses were conducted on the 40 young and 35 older participants. Median RTs were calculated in milliseconds (ms) for each individual in all conditions. Overall accuracy on all tasks was quite high (young participants: $M=97.25 \%$ correct; $S D=3.75$; old participants: $M=93 \%$ correct; $S D=14.01$ ). RTs and accuracy of responses in the different subtests are presented in Table 1. The effect of perceptual and motor conflict was measured by comparing performance (RTs) on each task involving conflict resolution (subtests 3,5 , and 6) to the mean RT performance on the two control tasks (subtests 1 and 4).

The independence of perceptual and motor inhibitory processes in young participants was assessed with a 2 (Perceptual Conflict: yes $v s$. no) $\times 2$ (Motor Conflict: yes vs. no) factorial ANOVA with repeated measures. The analysis revealed significant main effects of Perceptual $[F(1,39)=$ $256.02 ; p<.001 ;$ eta square $=0.87]$ and Motor $[F(1,39)=$ 23.16; $p<.001$; eta square $=0.37$ ] conflicts, indicating slower RTs in the presence of conflict. The interaction effect between perceptual and motor conflict conditions was not significant $[F(1,39)=1.16 ; p=.28$; eta square $=0.02]$ (see Figure 2). A similar analysis was performed in the group of older participants [factorial $2 \times 2$ ANOVA with repeated measures]. The analysis demonstrated significant main effects of perceptual $[F(1,34)=54.49 ; p<.0001$; eta square $=0.62]$ and motor $[F(1,34)=44.05 ; p<.001$; eta square $=0.56]$ conflicts, again indicating slower RTs in the presence of conflict. There was also a significant interaction between the two types of conflicts $[F(1,34)=5.71$; $p<.05$; eta square $=0.14]$, indicating that simultaneous confrontation with the two kinds of conflicts does not lead to a simply additive effect in older participants (see Figure 2).

To be sure that the presence of differential effects (additive $v s$. interactive) in young and older participants was not due to differences in speed of processing (SOP), we performed a 2 (Perceptual Conflict: yes vs. no) $\times 2$ (Motor Conflict: yes $v s$. no) factorial ANOVA with the group (young $v s$. older) as a between subject variable. Performance (RT) on subtest 2 was used as a covariate controlling for SOP. As predicted from the previous analyses, we observed a significant interaction between group and the presence/absence

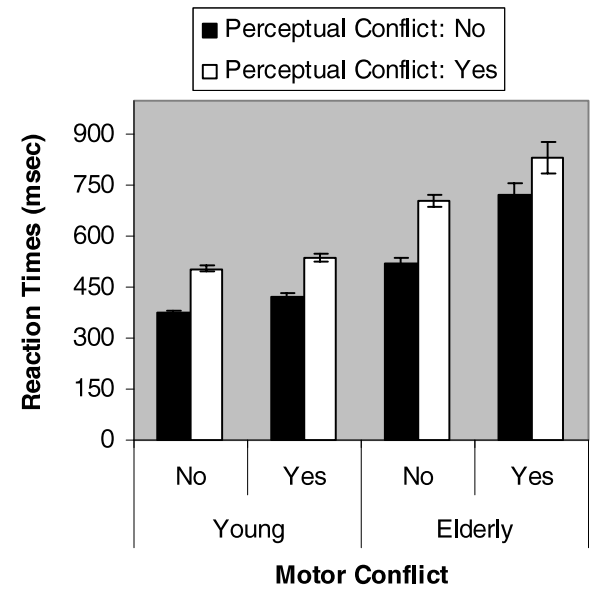

Fig. 2. Mean reaction times (ms) as a function of perceptual and motor conflict in young and elderly participants. Error bars represent standard errors. 
of perceptual $/$ motor conflicts $[F(1,72)=4.97 ; p<.05$; eta square $=0.07]$. Planned comparisons indicated the simultaneous confrontation to the two types of conflicts leads to an interactive effect in older participants $(p<.05)$ but not in young participants $(p>.05)$.

Finally, a correlational approach was used to determine the proportion of shared variance in young and older participants between RT performance on the perceptual and motor inhibitory tasks. Results indicate a larger proportion of shared variance between these tasks in older participants (perceptual and motor inhibition: young participants: $r=$ 0.18 , older participants: $r=0.47 ; p<.05)$.

We were also interested in investigating the existence of specific age-related differences on perceptual or motor inhibition. First, the performance of young and older participants was compared on subtests that required (subtest 3 ) or did not require (subtests 1 and 4) perceptual conflict resolution. A 2 (Group: young vs. older) $\times 2$ (Perceptual Conflict; yes vs. no) repeated measures ANOVA demonstrated main effects of Group $[F(1,73)=94.71 ; p<.0001$; eta square $=0.56$, with slower RTs in older participants] and Perceptual Conflict $[F(1,73)=548.16 ; p<.0001$; eta square $=0.88$, with slower RTs in the presence of conflict]. The interaction between Group and Perceptual Conflict was also significant $[F(1,73)=16.99 ; p<.001$; eta square $=$ $0.19]$. Planned comparisons demonstrated the presence of a perceptual conflict in both groups, but the conflict was greater for older participants (all $p<.001$ ). When SOP (RT on subtest 2) was taken as a covariate, the interaction effect remained significant $[F(1,72)=10.74 ; p<.005$; eta square $=$ $0.12]$.

The integrity of motor inhibitory processes in normal aging was then assessed with a 2 (Group: young vs. older) $\times$ 2 (Motor conflict: yes [subtest 5] vs. no [subtests 1 and 4]) repeated measures ANOVA. This analysis demonstrated significant main effects of Group $[F(1,73)=98.08 ; p<.0001$; eta square $=0.57$, with slower RTs in older] and Motor Conflict $[F(1,73)=128.89 ; p<.0001$; eta square $=0.64$, with slower RTs in the presence of conflict]. The interaction was also significant $[F(1,73)=51.74 ; p<.0001$; eta square $=0.41]$. Planned comparisons demonstrated the presence of a motor conflict in both groups, but again the conflict was larger for older participants (all $p<.001$ ). When RT on subtest 2 was taken as a covariate, the interaction effect remained significant $[F(1,72)=21.99 ; p<.0001$; eta square $=0.23$ ].

Finally, we wished to directly compare the size of perceptual and motor inhibitory effects in young and older participants. Inhibitory scores were built up by subtracting the performance obtained in the presence of conflict from the performance obtained in the no-conflict conditions, for the perceptual and motor conflict tasks, respectively [size of perceptual conflict: RT (subtest 3 - subtest 1 \& 4); size of motor conflict: RT (subtest 5 - subtest $1 \& 4$ ); see Table 1]. A 2 (Group: young vs. old) $\times 2$ (Conflict: perceptual vs. motor) repeated measures ANOVA demonstrated a main effect of Group $[F(1,73)=61.11 ; p<.0001$; eta square $=0.46$, with older participants presenting larger inhibitory effects $]$ and a main effect of Conflict $[F(1,73)=$ $7.19 ; p<.005$; eta square $=0.08$, with a larger inhibitory effect for perceptual conflict]. The interaction between Group and Conflict was also significant $[F(1,73)=18.18 ; p<$ .0001 ; eta square $=0.20]$. Planned comparisons revealed a smaller inhibitory effect in young participants for motor conflict in comparison to perceptual conflict $(p<.0001)$ but similar conflict effects in older participants $(p>.1)$. When RT on subtest 2 was taken as a covariate, the interaction effect remained significant $[F(1,72)=6.04 ; p<.05$; eta square $=0.07]$.

\section{DISCUSSION}

The aim of this study was twofold. First, we were interested in determining whether perceptual and motor inhibitory processes in normal aging are based on different cognitive resources, as was previously demonstrated in young participants (Nassauer \& Halperin, 2003). Second, we were interested in comparing the integrity of these two inhibitory processes in a single group of older participants. Indeed, very few earlier studies had been interested in evaluating the generality or specificity of the inhibitory dysfunction in normal aging by simultaneously administering several tasks assessing different inhibitory processes (Andrés et al., 2008; Belleville et al., 2006; Charlot \& Feyereisen, 2005; Kramer et al., 1994). In the present study, results indicate that, unlike young participants, healthy older participants seems to rely more on common cognitive resources necessary to resolve perceptual and motor conflicts (as indicated by ANOVA and correlation analyses). Furthermore, the effect of aging on inhibitory functioning was not explained by a slowing down of processing speed. Finally, the direct comparison of the two tasks indicates similar effect sizes for perceptual and motor conflict in older participants, but a larger effect size for perceptual conflict in young participants.

Nassauer and Halperin (2003) interpreted the presence of a merely additive effect when young participants have to simultaneously resolve motor and perceptual conflicts (in comparison to the resolution of each conflict in isolation) as indicating that the two inhibitory processes require separate cognitive resources. Indeed, according to the limited capacity resource model, if two co-occurring cognitive processes use the same resources, more than a simple additive increase in reaction time would be expected because the limited energy pool must be shared by both cognitive processes (Sergeant, 1996). The attribution of perceptual and motor conflict resolution processes to distinct cognitive resources in young participants is in agreement with theoretical frameworks that consider that inhibition is not unitary but consists in a series of different and specific processes (Harnishfeger, 1995; Kipp, 2005; Nigg, 2000). More specifically, the dissociation we observed here between perceptual and motor conflicts tasks fits particularly well with the Dempster and Corkill's (1999a,b) proposal that developmentally and functionally distinct inhibitory processes are 
associated with the perceptual and motor cognitive domains, as well as with the verbal domain (that was not explored in the present study).

Contrary to what was observed in young participants, the performance of older people was associated with an interactive effect when two conflicts have to be resolved simultaneously. According to the limited capacity resource model (Sergeant, 1996), this indicates that perceptual and motor conflicts share some cognitive resources and thus that these two inhibitory processes cannot be considered as independent and distinct processes in normal aging. The existence of an overlap in the cognitive resources needed to perform tasks that are considered to be independent in young participants can be interpreted as reflecting the presence of a dedifferentiation process in older people. The age-related dedifferentiation is supported by evidence showing increased correlations in normal aging among different cognitive tasks, or between sensorimotor and cognitive domain (e.g., Babcock et al., 1997; Baltes \& Lindenberger, 1997; Mitrushina \& Satz, 1991). Dedifferentiation was interpreted as a difficulty in recruiting specialized neural mechanisms during the performance of cognitive tasks (Li \& Lindenberger, 1999). This interpretation was supported by functional neuroimaging studies showing that age-related hemispheric asymmetry reductions in prefrontal or posterior activations during the performance of higher-order cognitive functions is associated to a lower performance (Grady et al., 2006; Park et al., 2004). In that context, it must be emphasized that further neuroimaging studies will be necessary to determine whether the performance on perceptual and motor inhibitory tasks is really more dependent on common cerebral areas in older than young participants. Such data will be the strongest argument in favor of the explanation of lower inhibitory abilities in normal aging as resulting from a dedifferentiation process.

The presence of a dedifferentiation process was previously found to be associated with less efficient cognitive functioning in various domains (Baltes \& Lindenberger, 1997; Lindenberger \& Baltes, 1997; for a review in the neuroimaging domain, see Cabeza, 2002). This association was also found in the present study because we observed that older participants performed worse on both the motor and perceptual conflict resolution tasks and performance on these tasks was more strongly correlated in older than younger participants. These results are consistent with previous studies that have shown impaired inhibitory abilities in normal aging (e.g., Butler et al., 1999; Hartley, 1993; May \& Hasher, 1998; Nielson et al., 2002; Spieler et al., 1996). Of interest, the inhibitory deficits were not found to be specific, because both motor and perceptual tasks were impaired in our older participants. An absence of specificity was also observed in another study (Belleville et al., 2006) that explored the inhibitory processes associated with the linguistic and perceptual domains using the Hayling (Burgess \& Shallice, 1996) and Stroop (Stroop, 1935) tasks, respectively. Taken as a whole, these results support the hypothesis that the inhibitory dysfunction associated with normal aging affects a whole range of cognitive domains.

However, other data indicate that not all inhibitory tasks are impaired in normal aging (Charlot \& Feyereisen, 2005; Kramer et al., 1994). More specifically, normal aging is characterized by an impairment of controlled/intentional inhibitory processes associated with a preservation of automatic/unintentional inhibitory processes (Andrés, 2008; Andrés et al., 2008; Collette et al., 2008b). It must, nevertheless, be emphasized that in the Belleville et al. (2006) study, as in the present one, all the inhibitory tasks administered can be considered to be controlled or intentional. Thus, the apparent widespread inhibitory dysfunction across cognitive domains may be related to the nature of the tasks used (controlled/intentional vs. automatic/unintentional). Bearing on this interpretation, we have recently obtained data showing a selective preservation of unintentional inhibitory processes associated with an impairment of intentional processes in the cognitive domains of working memory, semantic memory and episodic memory (Collette et al., 2008a).

Finally, it should be noted that the comparison of the size of the inhibitory effects for the two conflict resolution tasks demonstrated that the motor and perceptual tasks have a similar level of difficulty for older participants whereas young participants find it easier to resolve the motor conflict than the perceptual conflict. The presence of larger inhibitory effects in young participants for the perceptual than motor conflict resolution task cannot be explained by the external structures of the tasks administered (e.g., the kind of stimuli presented or the kind of response required) and may be interpreted as support for the dissociation between perceptual and motor inhibitory processes proposed by Dempster and Corkill $(1999 \mathrm{a}, \mathrm{b})$ and also by Nassauer and Halperin (2003). Although the presence of less efficient perceptual and motor inhibitory processes was frequently reported in normal aging (e.g., Butler et al., 1999; Hartley, 1993; Kramer et al., 1994; May \& Hasher, 1998; Nielson et al., 2002; Spieler et al., 1996), the direct comparison of the efficiency of these processes using very similar tasks has not yet been performed, to the best of our knowledge. The absence of a dissociation between perceptual and motor inhibitory effects in these participants is indicative of the presence of qualitative (and not only quantitative) changes in inhibitory functioning during normal aging. However, the interpretation of such changes remains speculative at this time. We tentatively propose that this absence of dissociation suggests the presence of a dedifferentiation process, leading to the involvement of common resources to perform tasks that are based on distinct resources in young participants. Obviously, confirmation with neuroimaging studies is needed to further support this interpretation.

To summarize, results of the current study indicate lower performance level and increased correlation among perceptual and motor inhibitory tasks in older compared with younger participants. Taken as a whole, these results are indicative 
that these tasks depend on distinct inhibitory processes in young adulthood but common cognitive resources with advancing age.

\section{ACKNOWLEDGMENTS}

This work was supported by the Belgian National Fund for Scientific Research (FNRS), the University of Liège and the Government of the French-Speaking Community of Belgium (Actions de Recherche Concertées, Grant 05-10-332). F. Collette is Senior Research Associate at the FNRS. All authors and author's institution disclose any actual or potential conflicts of interest including any financial, personal or other relationships with other people or organizations within three years of beginning the work submitted that could inappropriately influence (bias) their work. We acknowledge the editing assistance of Dr. Leslie Berkelhammer, under the auspices of the Research Editing Consultant Program of the International Neuropsychological Society International Liaison Committee.

\section{REFERENCES}

Andrés, P. (2008). Equivalent part set cuing effects in younger and older adults. European Journal of Cognitive Psychology, (in press).

Andrés, P., Guerrini, C., Phillips, L., \& Perfect, T. (2008). Differential effects of aging on executive and automatic inhibition. Developmental Neuropsychology, 33, 101-123.

Andrés, P. \& Van der Linden, M. (2000). Age related differences in supervisory attentional system functions. The Journals of Gerontology. Series B, Psychological Sciences and Social Sciences, 55, P373-P380.

Andrés, P., Van der Linden, M., \& Parmentier, F.B.R. (2004). Directed forgetting in working memory: Age-related differences. Memory, 12, 248-256.

Babcock, R.L., Laguna, K.D., \& Roesch, S.C. (1997). A comparison of the factor structure of processing speed for younger and older adults: Testing the assumption of measurement equivalence across age groups. Psychology and Aging, 12, 268-276.

Baltes, P.B. \& Lindenberger, U. (1997). Emergence of a powerful connection between sensory and cognitive functions across the adult life span: A new window to the study of cognitive aging? Psychology and Aging, 12, 12-21.

Belleville, S., Rouleau, N., \& Van der Linden, M. (2006). Use of the Hayling task to measure inhibition of prepotent responses in normal aging and Alzheimer's disease. Brain and Cognition, 62, 113-119.

Burgess, P.W. \& Shallice, T. (1996). Response suppression, initiation and strategy use following frontal lobe lesions. Neuropsychologia, 34, 263-273.

Butler, K.M., Zacks, R.T., \& Henderson, J.M. (1999). Suppression of reflexive saccades in younger and older adults: Age comparison on an antisaccade task. Memory and Cognition, 27, 584-591.

Cabeza, R. (2002). Hemispheric asymmetry reduction in old adults: The HAROLD Model. Psychology and Aging, 17, 85-100.

Charlot, V. \& Feyereisen, P. (2005). Mémoire épisodique et déficit d'inhibition au cours du vieillissement cognitif: un examen de l'hypothèse frontale. L'Année Psychologique, 105, 323-357.

Cohen, J. (1988). Statistical power analysis for the behavioral sciences. Hillsdale, NJ: Lawrence Erlbaum Associates.
Collette, F., Germain, S., Hogge, M., \& Van der Linden, M. (2008a). Inhibitory control of memory in normal aging: Dissociation between impaired intentional and preserved unintentional processes. (Submitted).

Collette, F., Schmidt, C., Scherrer, C., Adam, S., \& Salmon, E. (2008b). Specificity of inhibitory deficits in normal aging and Alzheimer's disease. Neurobiology of Aging, (Epub ahead of print).

Connelly, S.L. \& Hasher, L. (1993). Aging and the inhibition of spatial location. Journal of Experimental Psychology: Human Perception and Performance, 19, 1238-1250.

Connelly, S.L., Hasher, L., \& Zacks, R.T. (1991). Age and reading: The impact of distraction. Psychology and Aging, 6, 533-541.

Dempster, F.N. \& Corkill, A.J. (1999a). Individual differences in susceptibility to interference and general cognitive ability. Acta Psychologica, 101, 395-416.

Dempster, F.N. \& Corkill, A.J. (1999b). Interference and inhibition in cognition and behaviour: Unifying themes for educational psychology. Educational Psychology Review, 11, 1-88.

Dulaney, C.L. \& Rogers, W.A. (1994). Mechanisms underlying reduction in Stroop interference with practice for young and old adults. Journal of Experimental Psychology: Learning, Memory and Cognition, 20, 470-484.

Eenshuistra, R.M., Ridderinkhof, K.R., \& van der Molen, M.W. (2004). Age-related changes in antisaccade task performance: Inhibitory control or working-memory engagement? Brain and Cognition, 56, 177-188.

Grady, C.L., Springer, M.V., Hongwanishkul, D., McIntosh, A.R., \& Winocur, G. (2006). Age-related changes in brain activity across the adult lifespan. Journal of Cognitive Neuroscience, $18,227-241$.

Harnishfeger, K.K. (1995). The development of cognitive inhibition: Theories, definitions, and research evidence. In F. Dempster \& C. Brainerd (Eds.), New Perspectives on Interference and Inhibition in Cognition (pp. 175-204). New York: Academic Press.

Harnishfeger, K.K. \& Bjorklund, D.F. (1993). The ontogeny of inhibitory mechanisms: A renewed approach to cognitive development. In M.L. Howe \& R. Pasnak (Eds.), Emerging Themes in Cognitive Development. Vol. 1. Foundations (pp. 28-49). New York: Springer-Verlag.

Hartley, A.A. (1993). Evidence for selective preservation of spatial selective attention in old age. Psychology and Aging, 8, 371-379.

Hartley, A.A. \& Kieley, J.M. (1995). Adult age differences in the inhibition of return of visual attention. Psychology and Aging, 10, 670-684.

Hartman, M. \& Hasher, L. (1991). Aging and suppression: Memory for previously relevant information. Psychology and Aging, 6, 587-594.

Hasher, L., Stoltzfus, E.R., Zacks, R.T., \& Rypma, B. (1991). Age and inhibition. Journal of Experimental Psychology: Learning Memory and Cognition, 17, 163-169.

Hasher, L., Zacks, R.T., \& May, C.P. (1999). Inhibitory control, circadian arousal, and age. In D. Gopher \& A. Koriat (Eds.), Attention \& Performance, XVII, Cognitive Regulation of Performance: Interaction of Theory and Application (pp. 653675). Cambridge, MA: MIT Press.

Houx, P.J., Jolles, J., \& Vreeling, F.W. (1993). Stroop interference: Aging effects assessed with the Stroop Color-Word Test. Experimental Aging Research, 19, 209-224. 
Kane, M.J., Hasher, L., Stolzfuz, E.R., Zacks, R.T., \& Connelly, S.L. (1994). Inhibitory attentional mechanisms and aging. Psychology and Aging, 9, 103-112.

Kieley, J.M. \& Hartley, A.A. (1997). Age-related equivalence of identity suppression in the Stroop color-word task. Psychology and Aging, 12, 22-29.

Kipp, K. (2005). A developmental perspective on the measurement of cognitive deficits in attention-deficit/hyperactivity disorder. Biological Psychiatry, 57, 1256-1260.

Kramer, A.F., Humphrey, D.G., Larish, J.F., Logan, J.D., \& Strayer, D.L. (1994). Aging and inhibition: Beyond a unitary view of inhibitory processing in attention. Psychology and Aging, 9, 491-512.

Langley, L.K., Overmier, J.B., Knopman, D.S., \& Prod'Homme, M.S. (1998). Inhibition and habituation: Preserved mechanisms of attentional selection in aging and Alzheimer's disease. Neuropsychology, 12, 353-366.

Li, S.C., Huxhold, O., \& Schmiedek, F. (2004). Aging and attenuated processing robustness: Evidence from cognitive and sensorimotor functioning. Gerontology, 50, 28-34.

Li, S.C. \& Lindenberger, U. (1999). Cross-level unification: A computational exploration of the link between deterioration of neurotransmitter systems dedifferentiation of cognitive abilities in old age. In L.G. Nilsson \& H.J. Markowitsch (Eds.), Cognitive Neuroscience of Memory (pp. 103-146). Seattle: Hogrefe \& Huber.

Lindenberger, U. \& Baltes, E.B. (1997). Intellectual functioning in old and very old age: First results from the Berlin Aging Study. Psychology and Aging, 12, 410-432.

Lindenberger, U. \& Baltes, P.B. (1994). Sensory functioning and intelligence in old age: A strong connection. Psychology and Aging, 9, 339-355.

Mattis, S. (1976). Mental status examination for organic mental syndrome in the elderly patient. In L. Bellack \& T.B. Karasu (Eds.), Geriatric Psychiatry (pp. 77-121). New York: Grune \& Stratton.

May, C.P. \& Hasher, L. (1998). Synchrony effects in inhibitory control over thought and action. Journal of Experimental Psychology: Human Perception and Performance, 24, 363-379.

Mitrushina, M. \& Satz, P. (1991). Analysis of longitudinal covariance structures in assessment of stability of cognitive functions in elderly. Brain Dysfunction, 4, 163-173.

Monsch, A.U., Bondi, M.W., Salmon, D.P., Butters, N., Thal, L.J., Hansen, L.A., Wiederholt, W.C., Cahn, D.A., \& Klauber, M.R. (1995). Clinical validity of the Mattis dementia rating scale in detecting dementia of the Alzheimer type. Archives of Neurology, 52, 899-904.
Murphy, D.R., McDowd, J.M., \& Wilcox, K. (1999). Inhibition and aging: Similarities between younger and older as revealed by the processing of unattended auditory informations. Psychology and Aging, 14, 44-59.

Nassauer, K.W. \& Halperin, J.M. (2003). Dissociation of perceptual and motor inhibition processes through the use of novel computerized conflict tasks. Journal of the International Neuropsychological Society, 9, 25-30.

Nielson, K.A., Langeneker, S.A., \& Garavan, H. (2002). Differences in the functional neuroanatomy of inhibitory control across the adult lifespan. Psychology and Aging, 17, 56-71.

Nigg, J.T. (2000). On inhibition/disinhibition in developmental psychopathology: Views from cognitive and personality psychology and a working inhibition taxonomy. Psychological Bulletin, 126, 220-246.

Park, D.C., Polk, T.A., Park, R., Milnear, M., Savage, A., \& Smith, M.R. (2004). Aging reduces neural specialization in ventral visual cortex. Proceedings of the National Academy of Sciences of the United States of America, 101, 13091-13095.

Rush, B., Barch, D., \& Braver, T. (2006). Accounting for cognitive aging: Context processing, inhibition or processing speed. Aging, Neuropsychology and Cognition, 13, 588-610.

Schneider, W., Eschman, A., \& Zuccolotto, A. (2002). E-Prime User's Guide. Pittsburgh, PA: Psychology Software Tools Inc.

Sergeant, J. (1996). A theory of attention: An information processing perspective. In G.R. Lyon \& N.A. Krasnegor (Eds.), Attention, Memory and Executive Function (pp. 57-69). Baltimore: Paul H. Brookes Publishing Co.

Spieler, D.H., Balota, D.A., \& Faust, M.E. (1996). Stroop performance in healthy younger and older adults and in individuals with dementia of the Alzheimer's type. Journal of Experimental Psychology: Human Perception and Performance, 27, 461-479.

Stoltzfus, E.R., Hasher, L., Zacks, R.T., Ulivi, M., \& Goldstein, D. (1993). Investigations of inhibition and interference in younger and older adults. Journal of Gerontology, 48, P179-P188.

Stroop, J.R. (1935). Studies of interference in serial verbal reactions. Journal of Experimental Psychology, 6, 643-661.

Sullivan, M.P., Faust, M.F., \& Balota, D.A. (1995). Identity negative priming in older adults and individuals with dementia of the Alzheimer type. Neuropsychology, 9, 537-555.

Zacks, R.T., Hasher, L., \& Radvansky, G. (1996). Studies of directed forgetting in older adults. Journal of Experimental Psychology: Learning, Memory and Cognition, 22, 143-156. 